\title{
Metastasis of lung cancer through Batson's plexus: very rare but possible
}

\author{
Ragesh Panikkath MD, Saba Radhi MD, Sian Yik Lim MD, Manoj Thankam MD, \\ Frank Quattromani MD, Cynthia Ann Jumper MPH, MD
}

\begin{abstract}
Many malignancies, like prostate, colon, and breast cancer, metastasize through Batson's plexus (vertebral venous plexus). Although lung cancer is one of the most common cancers in the United States, its spread through Batson's plexus is considered extremely rare. We report a 76-year-old woman with adenocarcinoma of the lung who had metastasis affecting multiple contiguous vertebral bodies likely due to dissemination through Batson's plexus of veins. This plexus is a component of the cerebrospinal venous system (CSVS) consisting of the cranial venous system and the vertebral venous plexus (Batson's plexus). The CSVS is a valveless network of veins which are important in the venous drainage of the brain and spine. However, this venous network provides an easy channel for the dissemination of infections and malignant cells.
\end{abstract}

Key words: Lung cancer, Batson's plexus, metastasis, cerebrospinal venous system

\section{INTRODUCTION}

Metastases represent an important limitation in modern medicine in the treatment of cancer. One important channel for metastases involves the vertebral venous plexus, now named Batson's plexus. This valveless paravertebral network of veins can provide a channel for the spread of cancers of the colon, rectum, and prostate..$^{2-5}$ However, dissemination of lung cancer through this channel is considered extremely rare, probably because the entire pulmonary venous drainage and more than $85 \%$ of the bronchial circulation go to the left side of the heart through the pulmonary veins and do not have access to the cere-

Corresponding author: Ragesh Panikkath MD Contact Information: ragesh.panikkath@ttuhsc.edu DOI: 10.12746/swrccc2013.0104.046 brospinal venous system (CSVS). We report a patient with metastases from adenocarcinoma lung involving multiple contiguous vertebral bodies, most likely due to the spread through Batson's plexus.

\section{Case Presenttion}

A 76-year-old woman was referred for evaluation of an incidental lung mass. Magnetic resonance imaging and positron emission tomography - computed tomography (PET-CT) confirmed an isolated mass in the right lung. She underwent a thoracotomy with mediastinal and hilar lymphnode dissection, followed by lobectomy of the right upper lobe and segmentectomy of the right lower lobe. The pathology revealed a $3.5 \mathrm{~cm}$ poorly differentiated adenocarcinoma with vascular and lymph invasion. However, the margins were clear, and the lymph nodes were negative for malig- 
nancy. The tumor abutted the visceral pleura without invasion and was staged I B. The patient refused adjuvant chemotherapy. A follow up CT chest performed three months later revealed no new lung lesions, but showed multiple heterogeneous lesions in the right lobe of the liver. A liver biopsy showed only fibrosis. A year later, she had musculoskeletal pain in her upper back and shoulders and was treated for arthritis by her primary care doctor. A PET-CT scan showed lesions consistent with metastases in the bone, lung, liver, and adrenals (Figure 1). Involvement of multiple contiguous vertebral bodies, the pelvic bones, both clavicles and proximal humeri, and both femurs was noted. This distribution closely resembles the area of drainage of the Batson's plexus and supports this route for metastases. There were no metastases in bones below the elbows and knees. This distribution would not support an arterial route of spread in a patient with such widespread bony metastases. A biopsy from the hip confirmed metastatic adenocarcinoma from the lung. Tumor cells were positive for CK-7 and nuclear TTF1, negative for CK-20, EGFR wild type, and negative for the ENM4-ALK translocation. The

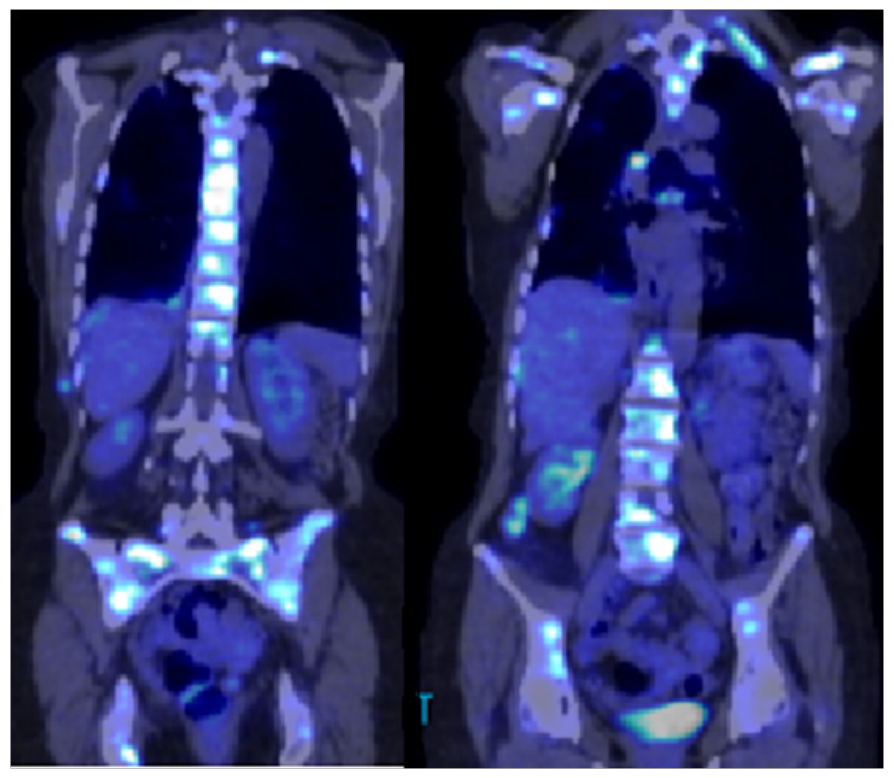

Figure 1. Fluoro PET CT scan showing metastases in multiple contiguous vertebral bodies and the pelvis suggesting spread through Batson's plexus. patient was offered chemotherapy with pemetrexed, carboplatin, becavizumab, and bisphosphonate for bony metastasis.

\section{Discussion}

Although veins leaving the vertebral bodies were noted as early as 1519 by Vesalius (founder of modern anatomy), ${ }^{1}$ Breschet (1819) and Batson (1940) provided a more complete description of the vertebral venous plexus (commonly known as Batson's plexus). ${ }^{1,6}$ This system extends from the sacrum to the cranium and forms a large capacitance venous system which communicates freely with the other venous systems through segmental vessels. This venous system is more correctly known as the cerebrospinal venous system since it consists of the cranial venous system (ophthalmic veins, cavernous sinus, dural veins, and intracranial veins) and the vertebral venous plexus. Together they form a continuous network of large capacity, plexiform, valveless veins which are important in the venous drainage of the head and spine.The vertebral venous (VVS) system consists of three intercommunicating divisions: 1 ) internal vertebral venous plexus within the spinal canal outside the dura, 2) external vertebral venous plexus outside the vertebral column, and 3) basivertebral veins which run horizontally within the vertebrae (Figures 2 and 3). The internal and external vertebral venous plexus consists of two anterior and two posterior veins and extends longitudinally along the spine from the basiocciput to the coccyx.

Flow in this valveless system of veins is bidirectional. It has an important role in the regulation of intracranial pressure with posture. ${ }^{1}$ The VVS communicates with the veins of the thoracoabdominal wall, veins of the back, azygous, cavae, veins of the pelvis, prostate, and sacral venous plexus. The CSVS provides a venous channel connecting the cerebral, abdominal, and pelvic cavities. In normal conditions, this system helps in venous drainage of the organs in these cavities. In cases of obstruction of the vena cava, the VVS provides an alternate channel bypassing blood from the area of its drainage. However, in 


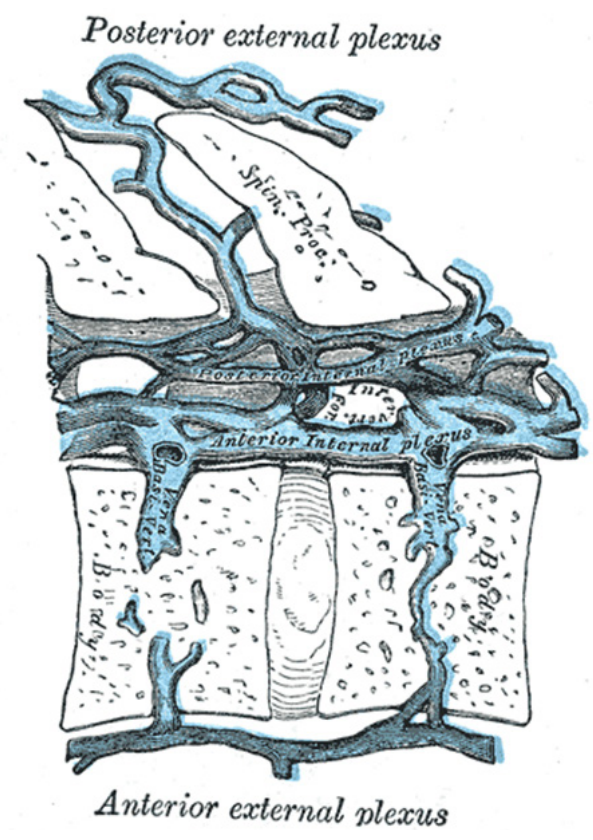

Figure 2. Diagram showing the vertebral venous plexus (image courtesy Wikipedia commons; http://en.wikipedia.org/wiki/File:Gray579.png; accessed 08/17/2013)

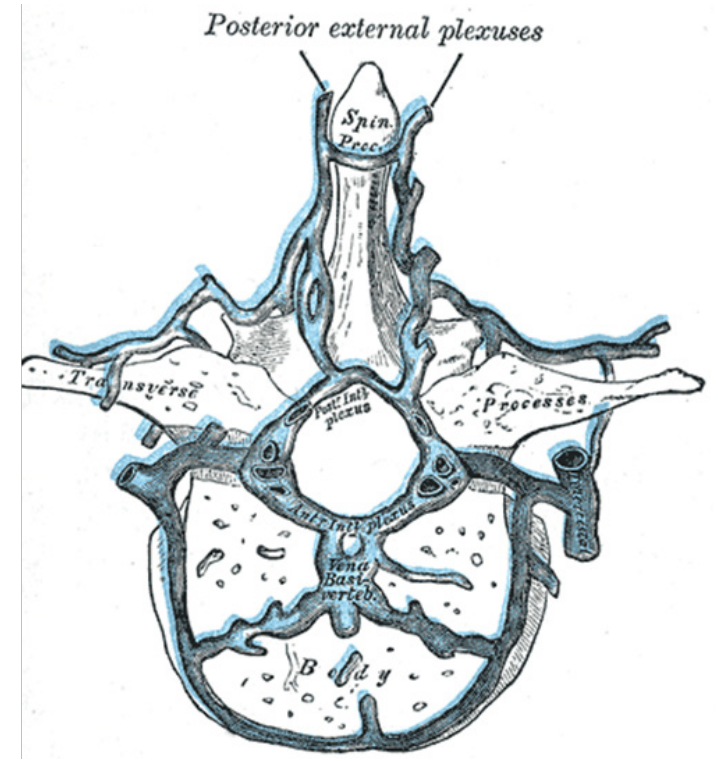

Figure 3. Diagram showing the vertebral venous plexus (image courtesy Wikipedia commons; http://en.wikipedia.org/wiki/File:Gray578.png; accessed 08/17/2013) pathologic conditions, the presence of this network of veins might be harmful. For example, the CSVS has been implicated in the spread of prostate cancer to the spine, brain, and the leptomeninges. ${ }^{1}$ This has been explained by direct communication of the prostatic venous plexus with the CSVS as demonstrated by Batson. "Experimentally, infusion of tumor cells in the tail veins of mice causes reproducible growth of tumors in the lumbar spine when the venae cavae have been occluded. ${ }^{8}$ Coman and Delong noted metastasis involving the vertebrae in $70 \%$ of mice when malignant cells were injected in femoral veins of rats with application of abdominal pressure. 'These experimental models suggest that tumors can spread through Batson's plexus. Venous air embolism during spinal surgery ${ }^{12}$ and dissemination of infections have also been attributed to the VVS. For example, infections of the urinary tract and after prostatic surgery can spread to the spine causing osteomyelitis. The CSVS has also been implicated in dissemination of intra-thoracic infections and parasitic infections to the brain. ${ }^{13}$

Breast, prostate, and lung cancers cause $80 \%$ of all bone metastases. Bone metastasis occurs in up to $66 \%$ of patients with cancer. Lung cancer commonly metastasizes to bone with autopsy evidence of bone metastases reported in $36 \%$ (range 30-55 $\%$ ) of patients. ${ }^{11}$ Adenocarcinoma and squamous cell carcinoma are the most common lung cancer histological types to involve bone. However, the spread of lung cancer in this manner through the CSVS is considered extremely rare. The characteristic of metastases spread through this system is the involvement of multiple contiguous vertebral bodies which would be extremely unusual through the arterial system, which spreads to the vertebral bodies through the nutrient arteries and affects noncontiguous vertebrae.

Lung cancer is inherently different from other cancers with respect to hematogenous metastases. Lung cancer usually spreads to the bone through direct arterial dissemination. This is because most of the blood return from the lungs (entire pulmonary blood flow and more than $85 \%$ of the bronchial circulation) 
drains into the pulmonary veins and in turn into the left side of the heart. However, about $13 \%$ of the blood from the bronchial arteries returns through the bronchial veins to reach the azygous and the accessory hemiazygous systems which communicate with Batson's plexus. The bronchial venous blood flow from the proximal airways (extra pulmonary) drains into the bronchial veins, whereas the bronchial venous flow from most of the lung parenchyma drains into the pulmonary vein and reaches the left atrium. The tumor cells from cancers (other than the lung) can reach the pulmonary capillaries through systemic veins and are retained in the lung. Hematogenous bone metastases from such cancers, in the absence of lung metastases, likely occur through Batson's plexus.

\section{Conclusion}

Batson's plexus of veins is a complicated valveless network of veins located near the vertebral column. In normal conditions, it provides venous drainage of the brain and spine; in pathological conditions, it allows dissemination of infections and cancer. However, the spread of lung cancer through this mechanism is considered extremely rare. We describe a patient with adenocarcinoma of the lung with metastasis to multiple contiguous vertebrae, which can only be explained by dissemination of cancer through Batson's plexus.

\section{KEYPOINTS}

1. The cerebrospinal venous system (CSVS) is a network of veins which provide venous drainage of the brain and spinal cord.

2. Infections and cancers, such as prostate cancer, can spread through this system.

3. Dissemination of lung cancer through the CSVS is extremely rare but possible due to dual venous return from the lung.
Author Affiliation: Ragesh Panikkath and Sian Lim are residents in Internal Medicine at TTUHSC, Lubbock, TX. Saba Radhi and Manoj Thankam are fellows in Medical Oncology at TTUHSC. Cynthia Jumper is a pulmonary physician in the Department of Internal Medicine at TTUHSC. Frank Quattromani is a radiologist at University Medical Center, Lubbock, TX.

Received: 08/17/2013

Accepted: 09/02/2013

Reviewers: Raed Alalawi MD, Lukman Tijani MD

Published electronically: 10/15/2013

Conflict of Interest Disclosures: None

\section{REFERENCES}

1. Nathoo N, Caris EC, Wiener JA, Mendel E. History of the vertebral venous plexus and the significant contributions of Breschet and Batson. Neurosurgery. 2011;69:1007-14; discussion 1014.

2. Onec B, Oksuzoglu B, Hatipoglu HG, Onec K, Azak A, Zengin N. Cavernous sinus syndrome caused by metastatic colon carcinoma. Clin Colorectal Cancer. 2007;6:593-6.

3. Mathew P, Fleming D, Adegboyega PA. Myelophthisis as a solitary manifestation of failure from rectal carcinoma. A Batson phenomenon? Arch Pathol Lab Med. 2000;124:1228-30.

4. Raymond PL, Balaa MA. Diplopia and diarrhea: ileal carcinoid metastatic to the central nervous system. Am J Gastroenterol. 1992;87:240-3.

5. Geldof AA. Models for cancer skeletal metastasis: a reappraisal of Batson's plexus. Anticancer Res. 1997;17:1535-9.

6. Batson OV. The Function of the Vertebral Veins and Their Role in the Spread of Metastases. Ann Surg. 1940;112:138-49.

7. Batson OV. The vertebral system of veins as a means for cancer dissemination. Prog Clin Cancer. 1967;3:1-18.

8. Nishijima Y, Uchida K, Koiso K, Nemoto R. Clinical significance of the vertebral vein in prostate cancer metastasis. $A d v \operatorname{Exp}$ Med Biol. 1992;324:93-100.

9. Coman DR, de LR. The role of the vertebral venous system in the metastasis of cancer to the spinal column; experiments with 
tumor-cell suspensions in rats and rabbits. Cancer. 1951;4:610-8. 10. Maccauro G, Spinelli MS, Mauro S, Perisano C, Graci C, Rosa MA. Physiopathology of spine metastasis. Int J Surg Oncol. 2011;2011:107969.

11. Coleman RE. Clinical features of metastatic bone disease and risk of skeletal morbidity. Clin Cancer Res. 2006;12:6243s6249 s.

12. Albin MS, Ritter RR, Pruett CE, Kalff K. Venous air embolism during lumbar laminectomy in the prone position: report of three cases. Anesth Analg. 1991;73:346-9.

13. Scrimgeour EM, Gajdusek DC. Involvement of the central nervous system in Schistosoma mansoni and S. haematobium infection. A review. Brain. 1985;108 ( Pt 4):1023-38. 